\title{
A study of the influence of the vegetation on the climatic conditions in an urban environment
}

\author{
S. Grignaffini \& A. Vallati \\ Department of Technical Physics, Rome University "La Sapienza”, Italy
}

\begin{abstract}
This work concerns the study of the variations of the climatic parameters in an urban area bordering a green area. The problem of the great urbanization of the territory led to the registration of high temperatures in the cities during the summer. Such a phenomenon, called a "heat island", is caused by the employment of those materials used for the urbanization of the territory, which have different responses to the solar radiation that influence the naked soil or on a soil with vegetation. Actually, the presence of a green area in a territory strongly urbanized changes its environmental parameters and those of a bordering zone. In particular, it is possible to record the variation of air and soil temperature and the variation of the wind speed and direction.

It has been studied in a typical urban context, in which a green area is surrounded by an urbanized area consisting of buildings, car parks and totally asphalted streets. Then the effect of different types of vegetation on the urban area climate was evaluated, with the help of numerical analysis, and, moreover, the influence area of a specific green area on the bordering urban context has been studied. The computational model employed is the software ENVI-MET, which successfully reproduces the interaction among urban surfaces, the vegetation and the atmosphere.

The outcomes achieved help to understand how a green area modifies a city climate, making an area climatically more benign both from the point of view of city inhabitants and also from the energetic point of view.

Keywords: vegetation, climatic condition, environment.
\end{abstract}




\section{Introduction}

Climate and the human standards of life have been negatively affected by the national territory urbanisation and, most of all, by extreme construction. In particular, urbanisation has worsened the problem of heat islands mainly because the vegetation density in the urban context has been reduced. As a matter of fact, many spaces which in the past were public green spaces are now paved to meet the needs of public car parking and those of the citizens. Often, even public courtyards and private gardens slowly leave the space to highly urbanised areas.

In Italy the situation related to the Municipal green areas in the 14 most important cities is extremely different. The per-capita availability is, on average, $15.6 \mathrm{~m}^{2}$ /inhab., with a maximum of $42.1 \mathrm{~m}^{2} /$ inhab. in Genoa and a minimum of $3.2 \mathrm{~m}^{2} /$ inhab. in Naples. Having seen the Ministerial Decree of 02/04/68 $\mathrm{n}^{\circ} 1444$ which envisages, for Municipalities with a population higher than 10.000 inhabitants, a minimum value of public green for sports and spare time, that is equipped green areas equal to $9 \mathrm{~m}^{2} /$ inhab, this values not reached by all the analysed cities analysed.

Local temperatures are affected by the decrease of green areas in the urban context.

This phenomenon has been studied under many different points of view. Rosenfeld et al. [1], in their studies related to the City of Los Angeles, have pointed out how, generally, the presence of asphalt in stead of vegetation and trees has heated the city by $3^{\circ} \mathrm{C}$ during the years.

A surface covered by vegetation has a lower radiation temperature compared to the temperature of another unanimated area of the same colour. The difference of maximum temperature can go beyond $20^{\circ} \mathrm{C}$. In the instance of great green areas, such as parks, vegetation modifies the air temperature of the area and this clearly, in the summer, improves the thermal conditions of the urban zone.

Other scholars have analysed how a park can vary the air temperature in the bordering urban network. Jauregui [2] in some of his evaluation of Mexico City verified that the presence of Chapultepec Park (500 ha) in terms of temperature variation has an impact in a $2 \mathrm{~km}$ distance area. More recent studies by $\mathrm{Ca}$ et al. [3] have pointed out how New Town's Central Park changes the environmental conditions in the surrounding area for a distance of $1 \mathrm{Km}$ in the direction where the wind usually has a greater intensity.

It can be asserted that in a micro scale the impact of vegetation on the environmental conditions is small, but still relevant. The results of other studies carried out by other scholars [4] have led to some important considerations. In particular, the impact zone of a green area depends on the extension of the green area itself and, most of all, on the distance between two bordering green areas. As a matter of fact, small green areas close to each other are preferable in comparison with one single green area of wide dimensions, in terms of variations of environmental conditions.

The parameter that influences the most the climatic variations in a green area is the shading which, obviously, inhibits the penetration of solar radiation. Other 
factors can have an impact on the temperature variation inside the green area and mostly in a neighbouring area, especially the geometry of he urban context.

This study focuses on the behaviour of a green area inside an urban zone, analysing the climatic conditions of the area itself and of the neighbouring one. Furthermore, the climatic impacts of a widening of a green area on the surrounding territory are evaluated and, most of all, what happens, if in stead of a green zone a paved car park is built. The software ENVI-MET developed at the Bochum University (Germany) [5], is used for the considerations at study. Such a system is often used by the scientific community to simulate the interactions amongst different urban surfaces, vegetation and atmosphere.

\section{Calculation pattern}

This study has been carried out with the software ENVI-MET which enables the analysis of effects on small scale of the different urban configurations, made of diverse geometrical features and coating materials, under different surrounding conditions. The pattern has been used to analyse the interactions between the urban environment and the atmosphere, considering as surrounding conditions those of the city of Rome.

The calculation pattern used implements a system of differential equations which are briefly mentioned below.

The system of basic equations used to describe the turbulent threedimensional flux is made of the Navier Stokes equations approximated to the Boussinesq formula, and of the continuity equation. The reference temperature of this system is $T_{r e f}$, that is the air temperature at a height of $z=2500 \mathrm{~m}$. Such a temperature represents, in fact, the average values of the conditions of the atmosphere within the meso scale. In the equations the constant term describes the velocity variation of the wind due to the resistance of the vegetal elements. The following equation can be used as the parameter of this outcome:

$$
S_{u(i)}=\frac{\overline{\partial p^{\prime}}}{\partial x_{i}}=c_{d, f} \cdot L A D(z) \cdot W \cdot u_{i}
$$

where p' is the local variation of pressure, $W=\left(u^{2}+v^{2}+w^{2}\right)^{0.5}$ is the average velocity of the air at the height $z$ and $L A D(z)$ is the density of the leaves of a plant of a certain height $z$. The mechanical resistance of a vegetal element $c_{d, f}$ is equal to 0.2 .

The distribution of temperature $(\vartheta)$ and of the specific humidity $(q)$ inside the atmosphere is the outcome of a system of equations:

$$
\begin{aligned}
& \frac{\partial \vartheta}{\partial t}+u_{i} \cdot \frac{\partial \vartheta}{\partial \chi_{i}}=K_{h} \cdot\left(\frac{\partial^{2} \vartheta}{\partial \chi_{i}^{2}}\right)+Q_{h} \\
& \frac{\partial q}{\partial t}+u_{i} \cdot \frac{\partial q}{\partial \chi_{i}}=K_{q} \cdot\left(\frac{\partial^{2} q}{\partial \chi_{i}^{2}}\right)+Q_{q}
\end{aligned}
$$


In a manner similar to the equations of the moment of the motion quantity, the two terms $\mathrm{Q}_{\mathrm{h}}$ and $\mathrm{Q}_{\mathrm{q}}$ are used to evaluate the heat and steam exchanged on the surface of a leaf with the atmospheric pattern.

As far as the turbulence pattern is concerned, the programme can choose among the $1^{\text {st }}$ order and the model 1.5 order implemented in the study by Mellor and Yamada [6] where two additional equations are inserted for the intensity of turbulence and dissipation rate.

The analysis of the radiation fluxes is extremely important in the mathematical patterns which face the issues of urban climate; as a matter of fact, the variation of these fluxes leads to the differences in the local climatic conditions. In ENVI MET 5 different absorption coefficients are used. These represent different radiation fluxes. Four of them are used to describe the impact of vegetation on direct and diffuse shortwave radiation, and in relation to the incoming and outgoing flux from the atmosphere generated by the long wave radiation. The LAI parameter is used in this coefficients. Such a parameter is the one-dimensional index of the vertical area of the leaf of a $z$ level plant at the summit of the plant. The last coefficient describes the obstruction in the diffusion of the radiation fluxes determined by the presence of construction buildings. Each point of the grind that constitutes the calculation pattern can be determined by these radiation fluxes.

Furthermore, different types of grounds and coating materials, that are typical of an urban context, can be simulated in this programme. In order to obtain a detailed simulation, the programme can allocate each cell certain thermodynamic and hydraulic features.

In the programme, vegetation in treated as a one-dimensional column of $\mathrm{z}_{\mathrm{p}}$ height constituted by a normalised leaf density (LAD) and by a normalised roots profile (RAD). This is a universal scheme which can be used for small plants and for big trees. The interaction among the leaves and the surrounding air can be expressed in terms of flux of direct heat $\left(J_{f, h}\right)$, flux of heat exchanged for evaporation $\left(J_{f, \text { evap }}\right)$ and flux for transpiration $\left(J_{f, \text { trans }}\right)$ :

$$
\begin{gathered}
J_{f, h}=1.1 \cdot r_{a}^{-1} \cdot\left(T_{f}-T_{a}\right) \\
J_{f, \text { evap }}=r_{a}^{-1} \cdot \Delta q \cdot \delta_{c} \cdot f_{w}+r_{a}^{-1} \cdot\left(1-\delta_{c}\right) \cdot \Delta q \\
J_{f, \text { trans }}=\delta_{c} \cdot\left(r_{a}+r_{s}\right)^{-1} \cdot\left(1-f_{w}\right) \cdot \Delta q
\end{gathered}
$$

where $T_{a}$ and $q_{a}$, are the temperature and the humidity of the surrounding air, $T_{f}$ is the temperature of leaves, while $r_{a}$ is the coefficient of thermal exchange between the leaf and the area, depending, obviously, on the geometry of the leaf and on the wind velocity. The exchange of heat for evaporation is controlled by the resistance of the leaf's stoma $\left(r_{s}\right)$, which changes in function of the type of plant, of the radiation of impact and of the amount $f$ water contained in the roots area.

The vegetation pattern is coupled with the atmosphere pattern using two terms: 


$$
\begin{gathered}
Q_{h}(z)=L A D(z) \cdot J_{f, h} \\
Q_{q}(z)=L A D(z) \cdot\left(J_{f, \text { evap }}+J_{f, \text { trans }}\right)
\end{gathered}
$$

The temperature of the soil and of the vertical surfaces is obtained solving the equations of energetic cost. For the soil's surface the temperature $T_{o}$ is given by:

$$
R_{s w, n e t}+R_{l w, \text { net }}-c_{p} \cdot \rho \cdot J_{h}^{o}-\rho \cdot L \cdot J_{q}^{o}-G=0
$$

where $R_{s w, \text { net }}$ and $R_{l w, n e t}$ are the net quantities of radiation (long wave and shortwave) absorbed by the surface of the ground and $G$ is the quantity of heat which is diffused by conduction inside the soil.

For the vertical surfaces, $G$ is substituted by the heat which penetrates inside the walls and it is obviously the function of the difference between the internal and external temperature and the transmittance of the wall.

The equations are solved for a three dimensional grind which's step can vary on the three dimensions $x, y$ and $z$. The Alternating Direction Implicit (ADI) method is applied to solve the system of differential equations. The pattern calculates the thermal transient with a step of 10 seconds.

\section{Outcomes}

The study has been carried out on an analysis area shown in Fig. 1 (SOL0).

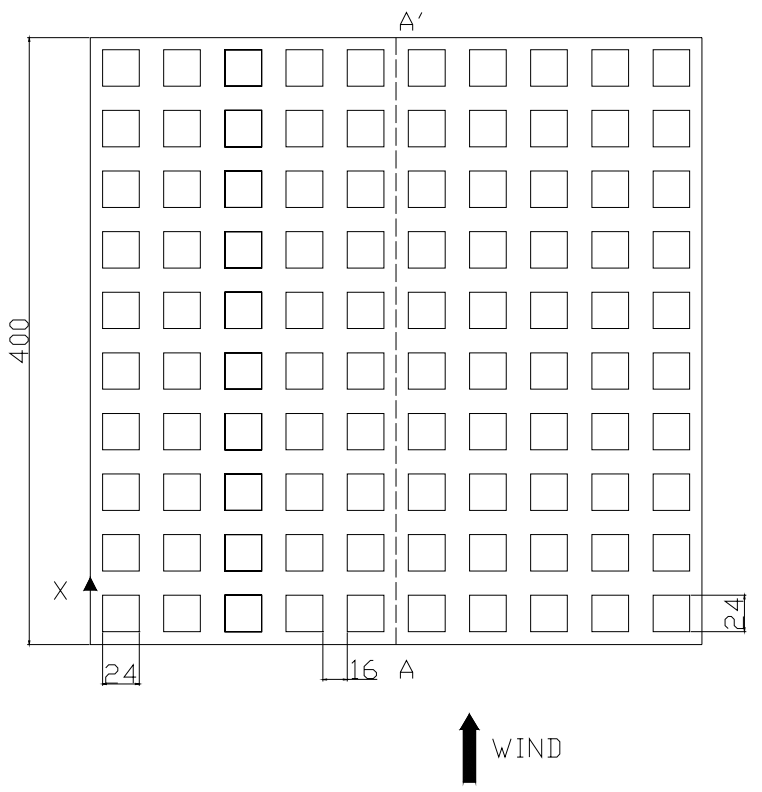

Figure 1: Map of the study area for the initial configuration (SOL0). 
Such a scheme implemented with the software ENVI-MET represents a classic situation identified inside an urban context. One could think of the network of four stored buildings at the centre of which an area was thought to be dedicated to the outdoor activities of the inhabitants of the surrounding area (Fig. 2).

For this study, a period of three days in July in Rome (July 14-16 2005) has been chosen. Such a period was characterised by high solar impact radiation values. The surrounding conditions of the pattern used are shown in Table 1.

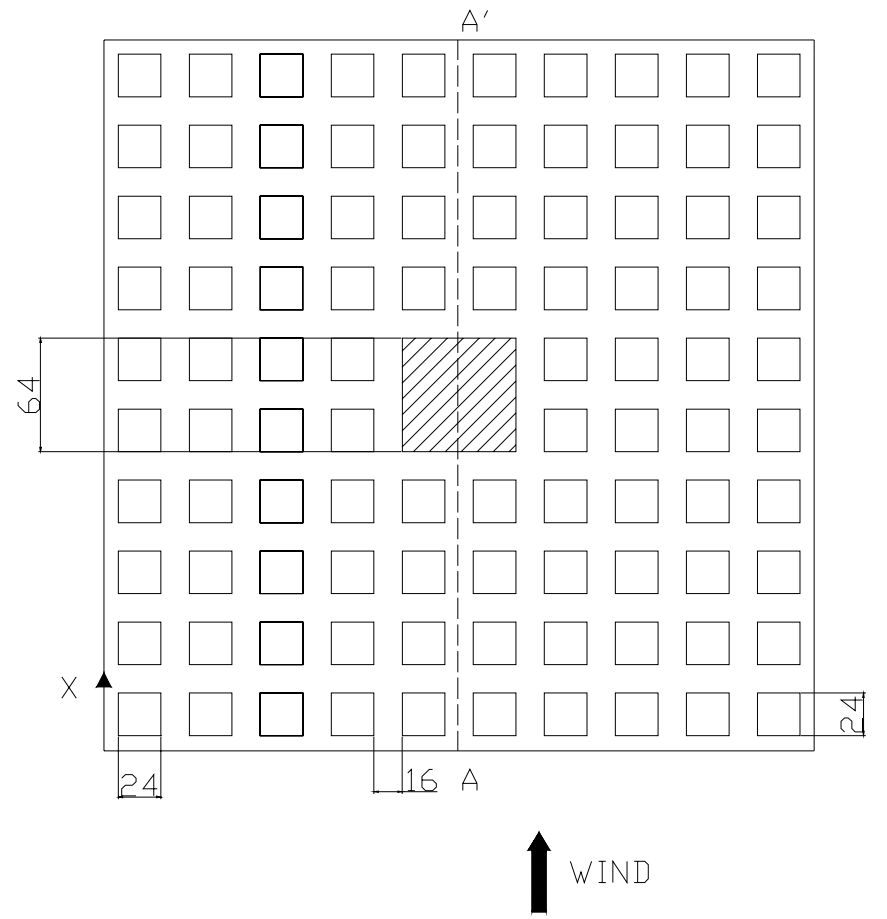

Figure 2: Map of the study area for the configuration with the central area differently coated.

Table 1: $\quad$ Input data used for the used mathematic pattern.

\begin{tabular}{|l|l|}
\hline Geographical coordinates & $\begin{array}{l}41^{\circ} 53^{\prime} 33,5^{\prime \prime} \\
12^{\circ} 29^{\prime} 31^{\prime \prime}\end{array}$ \\
\hline Calculation date and time & $14 / 07 / 200507.00$ A.M. \\
\hline Wind data & $($ South $)-(2 \mathrm{~m} / \mathrm{s})$ \\
\hline Grid dimensions & $130 \times 130 \times 130$ \\
\hline
\end{tabular}

In order to evaluate the effects on the urban climate of the type of coating used for the area in question, different types of alternatives were analysed: a grass-plot (SOL1), a garden planted with trees (SOL2) and an area with a water shine (SOL3). 
The surface of this area then changed widening its dimensions and reproposing the same solutions shown before for coating (Fig. 3).

In Table 2 the fundamental technical features of the three types of coating are shown:

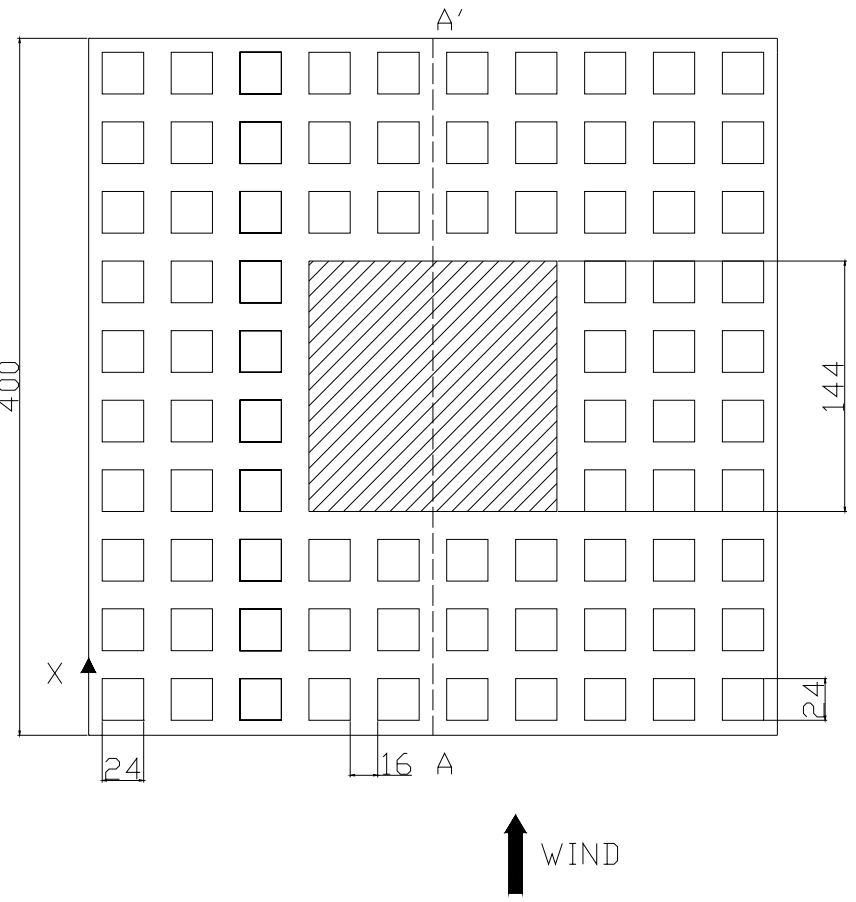

Figure 3: Map of the study area for the configuration of the central area widened and differently coated.

Table 2: Main physical data used for the three simulations that have been carried out.

\begin{tabular}{|l|c|c|c|c|}
\hline $\begin{array}{l}\text { Technical } \\
\text { characteristics }\end{array}$ & SOL0 & SOL1 & SOL2 & SOL3 \\
\hline RAD & - & 0.1 & 0.1 & - \\
\hline LAD & - & 0.3 & 2.18 & - \\
\hline Soil Temperature & 293 & 293 & 293 & 293 \\
\hline Tree height & - & - & $10 \mathrm{~m}$ & - \\
\hline Grass height & - & $0.1 \mathrm{~m}$ & - & - \\
\hline
\end{tabular}

This preliminary study has analysed the temperature of the soil, the temperature of the air at a height of $0.4 \mathrm{~m}$ and the temperature of the air at a height of $2 \mathrm{~m}$, in the intervention area and mostly in the surrounding area, as indexes for the evaluation of the urban climate. One o'clock p.m. has been chosen as reference time for the elaboration of the graphs which will be proposed later. 
Figure 4 shows the trend of the temperatures of the air at a height of $0.4 \mathrm{~m}$ from the soil for the first two configurations simulated for section (AA') chosen as parallel to the direction of the wind.

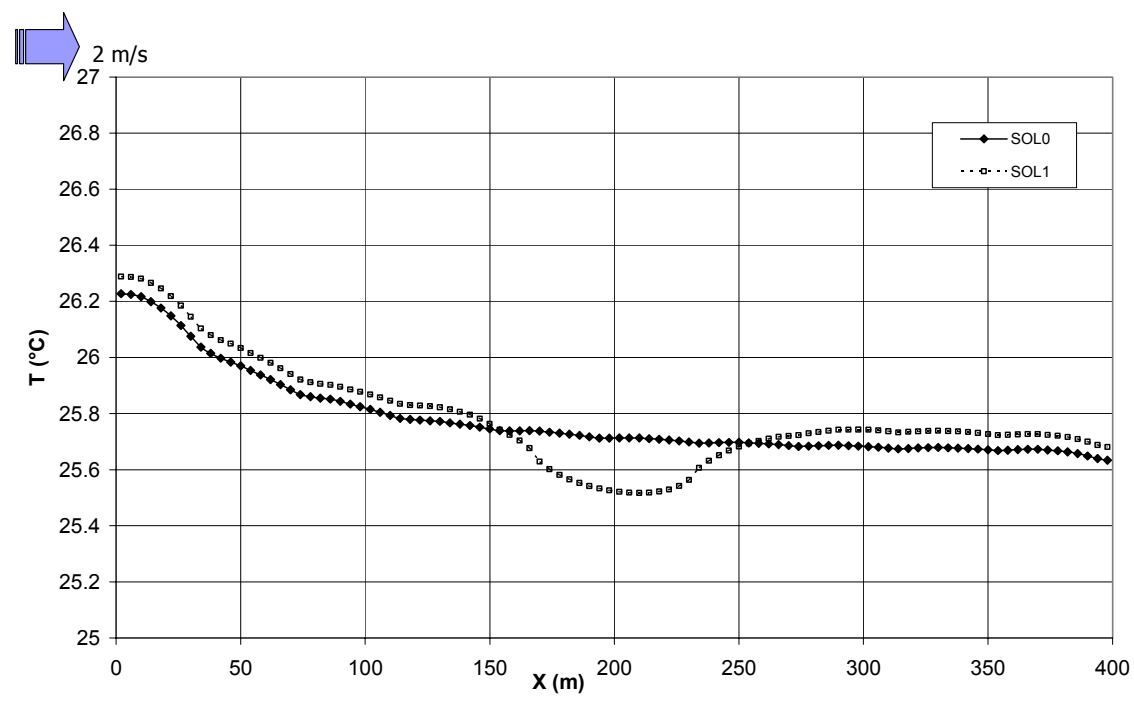

Figure 4: Trend of the air temperatures $(\mathrm{h}=0,4 \mathrm{~m})$ for the configurations SOL0 and SOL1.

From figure 4 one can notice how for a mild climate like the one proposed in this study (the temperature of the air at one o'clock p.m. is of about $25^{\circ} \mathrm{C}$ ), the temperature differences between a grass coating and an water proof coating are irrelevant and mostly how the temperature variation in an area bordering the green zone is very small.

Figure 5 shows the trend of the temperatures when trees are planted in addition to the lawn (SOL2) or an artificial water shine is built (SOL3).

Even in this case we can notice how, with relatively low temperatures during the summertime, the presence of different types of coating materials does not change the air temperature of the area itself and of a bordering area in a substantial way. It can be pointed out how the presence of trees (SOL 2) leads to a decrease of the air temperature by $0.5^{\circ} \mathrm{C}$ underneath the foliage $(\mathrm{h}=0.4 \mathrm{~m}$ from the soil), while in a neighbouring area, downwind the tree planted area, the temperatures are slightly lower with respect to the initial configuration for about 20-30 m. The trend of the temperature also highlights the importance of the wind in the analysis of urban climate. The $2 \mathrm{~m} / \mathrm{s}$ wind, which corresponds to about one knot, imposed by us for simulation, is considered to be a light breeze. Such a condition has clearly a small impact on the air temperature $f$ the downwind area.

Fig. 6 shows the trend of the temperature when the differently coated area is widened. In particular, the dimensions vary from $(64 \mathrm{~m} \mathrm{x} 64 \mathrm{~m})$ to $(144 \mathrm{~m} \mathrm{x}$ $144 \mathrm{~m})$. It can be noticed how the widening of an area coated with non 
impermeable materials produces a field of temperatures that improves the previous situation; in particular, the impact area of that zone sensibly increases especially when trees are planted and a three-lined area is created.

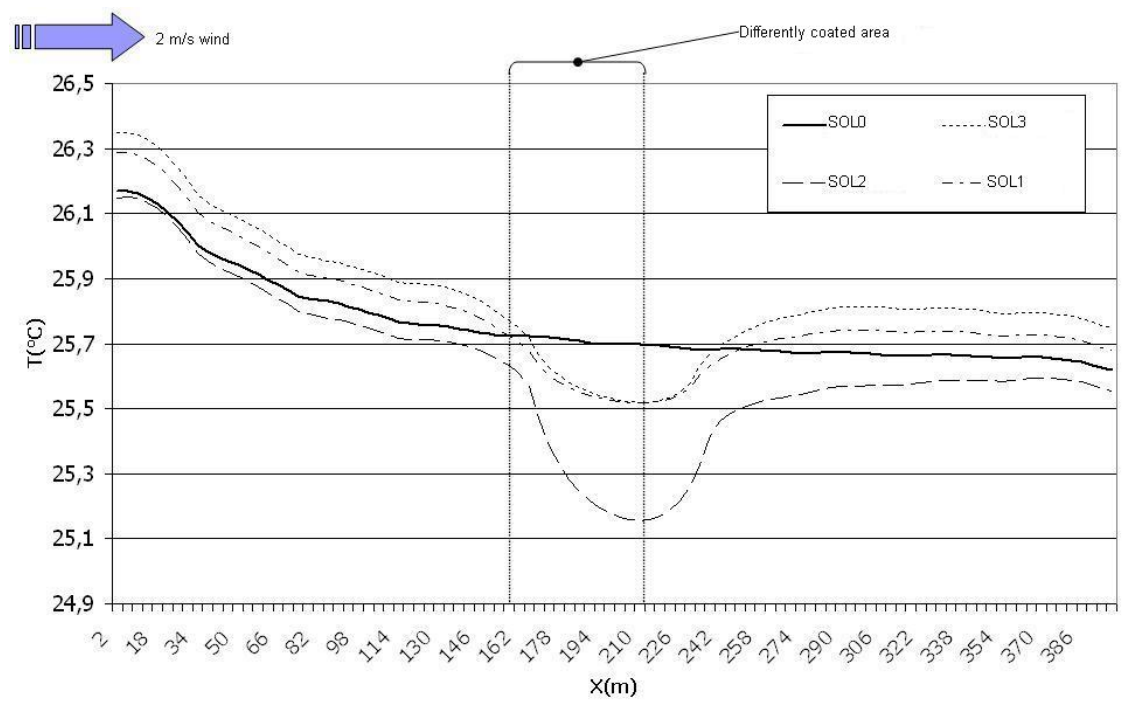

Figure 5: Trend of the air temperatures $(\mathrm{h}=0.4 \mathrm{~m})$ for the different configurations chosen for the purposes of this study.

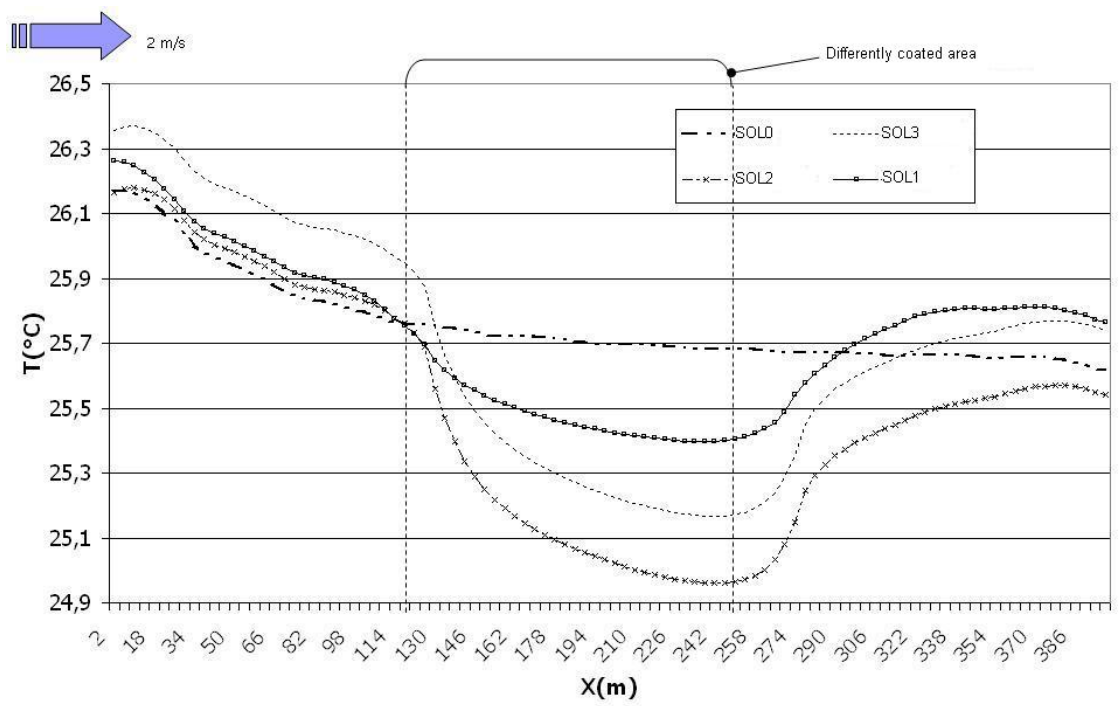

Figure 6: The trend of the air temperatures $(\mathrm{h}=0.4 \mathrm{~m})$ for the different configurations chosen for the purposes of this study. 
184 Sustainable Development and Planning III

Figs. 7 and 8, in stead, show the field temperature at the soil level for the different configurations and for the two different dimensions of the coated area.

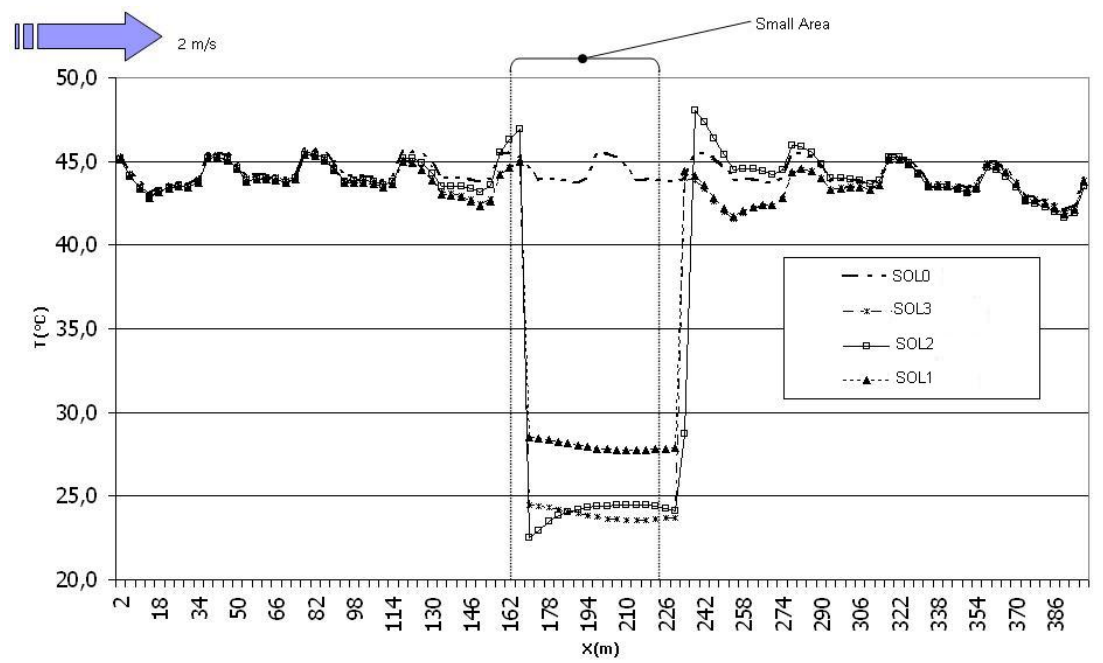

Figure 7: Trend of the soil temperatures for the different configurations chosen for the purposes of this study.

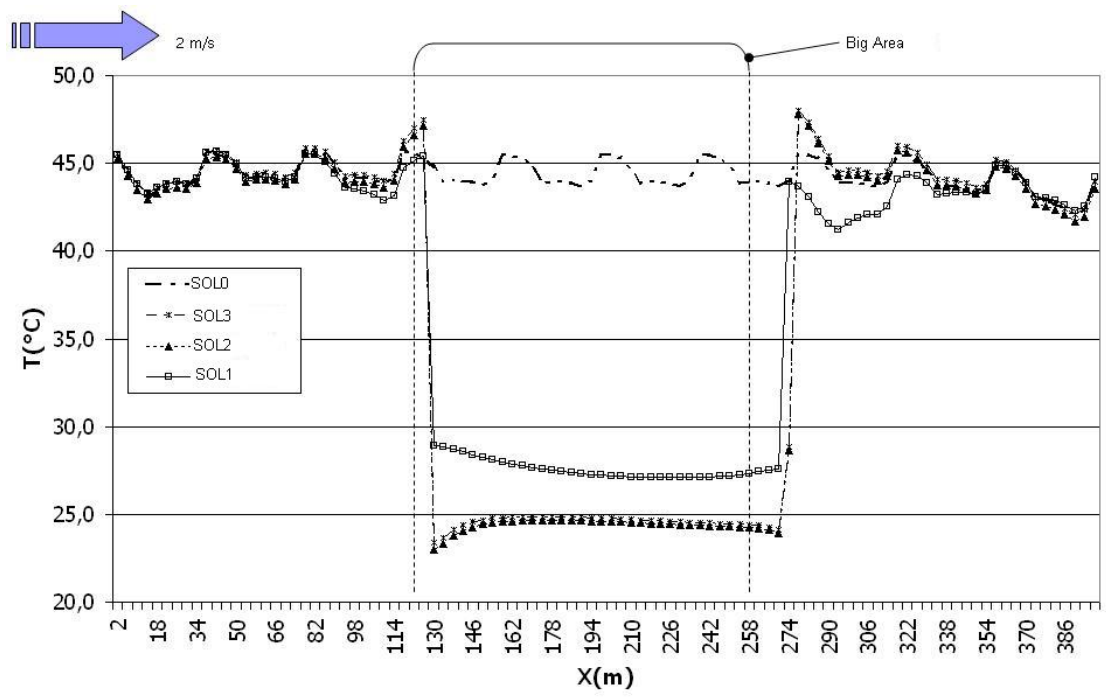

Figure 8: Trend of the soil temperatures for the different configurations chosen for the purposes of this study. 


\section{Conclusions}

The impact on the urban climate of an area coated with different impermeable materials has been evaluated in this study. The first results obtained with this software developed in Germany by Prof. M. Bruse, available on the net for the international research bodies, has led to good results, as was expected having seen the important applications in the literature that have used this study tool. In the future works we shall analyse more extreme summer conditions, characterised by higher external air temperatures and, mostly, verifying how a stronger wind can improve the conditions of the urban climate.

\section{References}

[1] A.H. Rosenfeld, H. Akbari, S. Bretz, B.L. Fishman, D.M. Kurn, D. Sailor, H. Taha, Mitigation of urban heat islands: materials, utility programs, updates, Journal of Energy and Buildings 22 1995 255-265.

[2] Jauregui, Influence of a large urban park on temperature and convective precipitation in a tropical city, Journal of Energy and Buildings 15-16 $1990457-463$.

[3] V.T. Ca, T. Asaeda, E.M. Abu, Reduction in air conditioning energy caused by a nearby park, Journal of Energy and Buildings 29 1998 83-92.

[4] T. Honjo, T. Takakura, Simulation of thermal effects of urban green areas on their surrounding areas, Journal of Energy and Buildings. 15-16 1990 443-446.

[5] Bruse M. ENVI-met homepage: www.envi-met.com, 2004.

[6] Mellor G. L. and T. Yamada (1975). A simulation of the Wangara atmospheric boundary layer data, J. Atmos. Sci. 32, 2309- 2329.

[7] Mayer H, Ho"ppe P. Thermal comfort of man in different urban environments. Theoretical and Applied Climatology 1987;38:43-9.

[8] Bruse, M. and H. Fleer (1998). Simulating Surface- Plant-Air Interactions Inside Urban Environments with a Three Dimensional Numerical Model, Environmental Software and Modelling, (13), S. 373-384. 